Pesq. Vet. Bras. 31(3):213-218, março 2011

\title{
Intoxicação espontânea e experimental por folhas e frutos de Erythroxylum deciduum (cocão) em ovinos no Estado de Santa Catarina ${ }^{1}$
}

\author{
Vanessa Borelli2, Daniela Lentz ${ }^{2}$, Luciane O.Veronezi², Tiffany C. Emmerich \\ da Silva², Leonardo Kaufer ${ }^{3}$, Sandra D. Traverso ${ }^{2}$ e Aldo Gava ${ }^{2}$
}

\begin{abstract}
Borelli V., Lentz D., Veronezi L.O., Silva T.C.E., Kaufer L., Traverso S.D. \& Gava A. 2011. [Spontaneous and experimental poisoning by the leaves and fruits of Erythroxylum deciduum (Cocão) in sheep in the State of Santa Catarina, Brazil.] Intoxicação espontânea e experimental por folhas e frutos de Erythroxylum deciduum (cocão) em ovinos no Estado de Santa Catarina. Pesquisa Veterinária Brasileira 31(3):213218. Departamento de Medicina Veterinária, Centro de Ciências Agroveterinárias, Universidade do Estado de Santa Catarina, Lages, SC 88520-000, Brazil. E-mail: a2ag@ cav.udesc.br

A disease in sheep with seasonal occurrence in the municipalities of Rancho Queimado and Ponte Alta do Sul, State of Santa Catarina, characterized by nervous manifestations and rapid death is described. The disease was reproduced experimentally in sheep giving the leaves of Erythroxylum deciduum collected in summer, at lethal doses from $10 \mathrm{~g} / \mathrm{kg}$ on. In one experiment in sheep with the fruits, the dose of $15 \mathrm{~g} / \mathrm{kg}$ was lethal. The onset of symptoms occurred within 2 hours after administration of the leaves, and the poisoning had a course from 10 minutes to 18 hours. Doses up to $20 \mathrm{~g} / \mathrm{kg}$ of leaves collected in winter caused slight signs of poisoning in two sheep. In the experiments, the clinical signs were depression and somnolence, and when the animals were moved, motor incoordination, imbalance, falls, difficulty to get up, and station with open legs; intense salivation, regurgitation, bruxism, increased heart rate and breathing was also observed. The clinical signs and death were exacerbated when the sheep were moved. The animals that died by the spontaneous and experimental poisoning did not show important gross lesions or microscopic alterations. It was concluded that the cut of the tree, allows growth of sprouts from the stems, encouraging the sheep to eat the plant. This fact and the availability of ripe fruits that fall off the trees can be made responsible for the poisoning with nervous signs of sheep, which occurs during the summer months in the state of Santa Catarina.
\end{abstract}

INDEX TERMS: Poisonous plants, Erythroxylum deciduum, "cocão", Erythroxylaceae, plant poisoning, sheep.

RESUMO.- Descreve-se uma enfermidade em ovinos de ocorrência sazonal, caracterizada por manifestações nervosas e morte rápida, nos municípios de Rancho Queimado

\footnotetext{
${ }^{1}$ Recebido em 1 de setembro de 2010.

Aceito para publicação em 22 de setembro de 2010.

${ }^{2}$ Laboratório de Patologia Animal, Departamento de Medicina Veterinária, Centro de Ciências Agroveterinárias, Universidade do Estado de Santa Catarina (UDESC), Av. Luiz de Camões 2090, Bairro Conta Dinheiro, Lages, SC 88520-000, Brasil. *Autor para correspondência: a2ag@cav.udesc.br

${ }^{3}$ Veterinário autônomo, Rua Germano Käufer Terra Nova 35, Bairro Taquaras, Município Rancho Queimado, SC 8847000, Brasil.
}

e Ponte Alta do Sul, no estado de Santa Catarina. A doença foi reproduzida experimentalmente em ovinos com a administração de folhas de Erythroxylum deciduum coletados durante o verão, com doses letais a partir de $10 \mathrm{~g} / \mathrm{kg}$ de peso vivo. Em um único experimento com os frutos a dose de $15 \mathrm{~g} / \mathrm{kg}$ foi letal. O início da manifestação dos sinais clínicos, ocorreu entre 0 e 2 horas após a administração das folhas ou dos frutos e a evolução da doença foi de 10 minutos a 18 horas. Doses de até $20 \mathrm{~g} / \mathrm{kg}$ de folhas coletadas no período de inverno, produziram alterações clínicas discretas em dois ovinos. Os sinais clínicos foram depressão, sonolência e, quando os animais eram movimentados, in- 
coordenação motora, desequilíbrio, quedas, dificuldade para se levantar e estação com os membros abertos. Também foi observado: salivação intensa, regurgitação, bruxismo, aumento das frequências cardíaca e respiratória. Os sinais clínicos se agravavam quando os animais eram movimentados e geralmente resultavam em morte. Os animais que morreram pelas intoxicações espontânea e experimental, não tinham alterações macroscópicas e microscópicas significativas. $O$ corte das árvores de $E$. deciduum faz com que o tronco rebrote, favorecendo a ingestão da planta pelos ovinos. Este fato e a disponibilidade de frutos maduros que caem ao chão podem ser responsabilizados pela doença de ovinos com sinais nervosos e ausência de lesões macroscópicas e microscópicas, que ocorrem nos meses de verão, no Estado de Santa Catarina.

TERMOS DE INDEXAÇÃO: Plantas tóxicas, Erythroxylum deciduum, cocão, Erythroxylaceae, intoxicação por planta, ovinos.

\section{INTRODUÇÃO}

A ocorrência de uma enfermidade de ovinos com morte rápida e sem apresentar lesões macroscópicas e microscópicas foi observada nos municípios de Rancho Queimado e Ponte Alta do Sul, Santa Catarina. Alguns criadores da região relacionavam a doença ao consumo de frutos de uma árvore conhecida como "cocão" (Erythroxylum deciduum), cuja toxidez foi descrita anteriormente por Colodel et al. (2004) e Barros et al. (2004). No entanto, a doença foi constatada também em propriedades onde havia grande quantidade de planta de E. deciduum, mas fora da época de frutificação.

E. deciduum St. Hil. é uma árvore conhecida popularmente como cocão, baga de pomba, fruta de pomba, que mede de 4 a 8 metros de altura (Lorenzi 2002). Seu habitat é diversificado podendo ser encontrado em beira de matas, beira de rios, regiões alagadiças, em campos e em encostas de morros (Sobral 1987). As folhas são simples, alternadas e com bordos inteiros. As flores são amareladas e os frutos tipo drupa (carnosos com uma única semente) que, quando maduros, são vermelhos. O florescimento começa no mês de agosto e os frutos amadurecem de outubro a janeiro (Lorenzi 2002).

O princípio ativo que atua na patogenia da doença ainda não é conhecido. Zuanazzi et al. (2001) detectaram uma gama de alcalóides tropanos em $E$. argentinum e $E$. deciduum. Dentre eles $3 \beta, 6 \beta$-ditigloyloxynortropano, 3 $\beta, 7 \beta$-ditigloyloxynortropano,4-hydroxyhygrinic ácido, methylecgonidina e tropacocaina. Resquício de cocaína foi isolado em E. deciduum (Aynilian et al. 1974). No entanto, a presença de cocaína nesta espécie foi discordada por Zuanazzi et al. (2001), pois detectaram um novo alcalóide que pode ter sido confundido com a cocaína, em outros estudos.

Na região sul do Brasil, são descritas mortes de ovinos com alterações neurológicas causadas pela ingestão de frutos da árvore Erythroxylum deciduum (Colodel et al. 2004) e Erythroxylum argentinum (Barros et al. 2004), ambas co- nhecidas popularmente como "cocão". A doença é descrita, em ovinos mantidos sob pastejo, em áreas que contém mata ciliar com grande quantidade da árvore em frutificação, cujos frutos caídos são consumidos com avidez (Barros et al. 2004, Colodel et al. 2004). Os surtos que ocorrem nos meses da frutificação do "cocão", se caracterizam por grandes perdas de ovinos, com uma taxa de morbidade de $31,8 \%$ e letalidade de $25,5 \%$ (Barros et al. 2004).

Os ovinos apresentam sinais clínicos predominantemente neurológicos como dificuldade para acompanhar o rebanho, andar lento com os membros rijos (Barros et al. 2004, Colodel et al. 2004), arrastam as pinças, se deparam contra objetos, permanecem em posição de cavalete com os membros abertos, apresentam tremores (Barros et al. 2004), tem quedas, ficam em decúbito lateral com tetania e cabeça voltada para trás, além de apresentar respiração abdominal, salivação intensa e cianose (Colodel et al. 2004). Os sinais clínicos são agravados quando os animais são movimentados. Em contra partida, foi observado que, quando os animais são calmamente retirados das pastagens e mantidos em local sem movimentação, ocorre uma diminuição no número de mortes (Barros et al. 2004, Colodel et al. 2004).

Os achados macroscópicos são inespecíficos e muitas vezes inexistentes, tal como: mucosas cianóticas, distenção da bexiga (Barros et al. 2004), edema pulmonar acentuado e hemorragia nas serosas, principalmente na pleura visceral (Colodel et al. 2004). Sementes e pedaços de frutos são encontrados nos pré-estômagos dos animais intoxicados (Barros et al. 2004, Colodel et al. 2004).

Com o objetivo de avaliar os aspectos epidemiológicos, clínicos e lesionais desta enfermidade em ovinos foram conduzidos experimentos nesta espécie com folhas e frutos de E. deciduum.

\section{MATERIAL E MÉTODOS}

\section{Doença espontânea}

Levantamento de históricos sobre ocorrência de doença em ovinos, com sinais nervosos e morte rápida, foi efetuado nos municípios de Rancho Queimado, localizado na região do Alto Vale do Itajaí e Ponte Alta do Sul, localizado no Planalto Catarinense. Dois surtos que ocorreram no município de Rancho Queimado foram avaliados clinicamente. Dois ovinos que morreram da enfermidade foram necropsiados e amostras de tecidos foram coletados para exame histopatológico.

\section{Intoxicação experimental ${ }^{4}$}

Para experimentação foram coletadas folhas maduras e jovens de árvores adultas e folhas de brotos de troncos anteriormente cortados, de Erythroxylum deciduum, em diferentes épocas do ano. As folhas foram mantidas em câmara fria e administradas por via oral em dose única para 15 ovinos, todos provenientes de propriedades livres de E. deciduum. Também foram coletados frutos maduros, os quais foram triturados e administrados por sonda para um ovino.

\footnotetext{
${ }^{4}$ Projeto de pesquisa aprovado no comitê de ética de Experimentação animal da Universidade do Estado de Santa Catarina (CAV/ UDESC). Protocolo 1.10/06 em 28.4.06.
} 
Quadro1. Delineamento da intoxicação experimental em ovinos com folhas e frutos de Erythroxylum deciduum

\begin{tabular}{|c|c|c|c|c|c|c|}
\hline Animal & Data & $\begin{array}{l}\text { Estágio das } \\
\text { folhas e frutos }\end{array}$ & $\begin{array}{l}\text { Peso } \\
\text { (k) }\end{array}$ & $\begin{array}{l}\text { Dose } \\
(\mathrm{g} / \mathrm{kg})\end{array}$ & $\begin{array}{l}\text { Total } \\
(\mathrm{g})\end{array}$ & Evolução \\
\hline Ovino 1 & Março de 2006 & Brotação de tronco & 40 & 10 & 400 & 12h* Morte \\
\hline Ovino 2 & Agosto de 2007 & Folhas adultas & 25 & 5 & 125 & Não adoeceu \\
\hline Ovino 3 & & Folhas adultas & 59 & 7,5 & 410 & Não adoeceu \\
\hline Ovino 4 & & Folhas adultas & 40 & 10 & 400 & Não adoeceu \\
\hline Ovino 5 & Outubro 2007 & Folhas jovens & 40 & 5 & 200 & Não adoeceu \\
\hline Ovino 6 & & Folhas jovens & 36 & 20 & 750 & Não adoeceu \\
\hline Ovino 7 & Março de 2008 & Folhas jovens & 42 & 15 & 630 & 3h Morte \\
\hline Ovino 8 & & Folhas jovens & 46 & 15 & 920 & $12 \mathrm{~h}$ Morte \\
\hline Ovino 9 & & Folhas jovens & 42 & 10 & 420 & Recuperou-se \\
\hline Ovino 10 & & Folhas jovens & 46 & 15 & 690 & 10 min Morte \\
\hline Ovino 11 & Junho de 2008 & Folhas adultas & 60 & 15 & 900 & Recuperou-se \\
\hline Ovino 12 & & Folhas adultas & 50 & 15 & 750 & Não adoeceu \\
\hline Ovino 13 & Setembro de 2008 & Folhas adultas & 37,5 & 15 & 570 & Não adoeceu \\
\hline Ovino 14 & & Folhas adultas & 39 & 20 & 800 & Não adoeceu \\
\hline Ovino 15 & Dezembro de 2008 & Brotação de tronco & 47 & 15 & 710 & 18h Morte \\
\hline Ovino 16 & Janeiro de 2009 & Frutos maduros & 60 & 15 & 900 & 30 min Morte \\
\hline
\end{tabular}

* Encontrado morto sem observação dos sinais clínicos.

Os ovinos foram mantidos em baias de alvenaria, alimentados com capim quicuio (Pennisetum clandestinum) e azevém (Lolium multiflorum) e água ad libitum. Todos os animais foram submetidos a exames clínicos, antes e, repetidas vezes, após a administração da planta e observados até a morte ou, recuperação. Exame eletrocardiográfico foi realizado em um ovino antes e após o fornecimento da planta. Os animais que morreram foram necropsiados e fragmentos de coração, pulmão, rumem, omaso, abomaso, intestino, fígado, rim, baço, linfonodo, músculo esquelético, glândula salivar, pâncreas, tireóide e sistema nervoso central, foram coletados para exame histológi$\mathrm{co}$, fixados em formalina a $10 \%$, processados rotineiramente, corados pela técnica de Hematoxilina-Eosina (HE) (Prophet et al. 1992) e observados no microscópio óptico.

O estudo foi realizado nas dependências do Laboratório de Patologia Animal, da Universidade do Estado de Santa Catarina. Amostras da planta foram coletadas nas propriedades em que ocorreu a doença e enviadas ao departamento de botânica da Universidade do Estado de Santa Catarina para identificação. O delineamento do experimento com folhas verdes e frutos de E. deciduum está representado no Quadro 1.

\section{Intoxicação espontânea}

\section{RESULTADOS}

Criadores de ovinos do município de Rancho Queimado e Ponte Alta do Sul, no estado de Santa Catarina, informaram sobre uma doença em ovinos, com manifestação nervosa e morte rápida, que ocorre anualmente no final da primavera e verão.

Em Ponte Alta do Sul, produtores informaram que durante muitos anos, nos meses de janeiro e fevereiro, ocorriam mortes de ovelhas e relacionaram a presença dos frutos maduros de "cocão" caídos junto ao pasto. Um proprietário informou que alguns animais consumiam os frutos maduros e, posteriormente, eram encontrados deitados e salivando, sendo que a morte ocorria entre 6 e 24 horas após o início dos sinais clínicos. De acordo com informações a mortalidade era variável chegando a $25 \%$ do rebanho. Devido às grandes perdas neste período do ano alguns produtores, desisti- ram da criação de ovinos. No município de Rancho Queimado, o histórico de morte de ovinos com sinais nervosos, foi relatado por três proprietários, porém, nenhum suspeitava da doença estar relacionada à ingestão do "cocão". Neste município foram acompanhados dois surtos (Surto 1 e 2). Em ambas as propriedades foram encontradas árvores de Erythroxylum deciduum (Fig.1A,B). Na propriedade em que ocorreu o Surto 1, a doença iniciou no mês de janeiro de 2006, quando $E$. deciduum encontrava- se com grande quantidades de frutos maduros (Fig.1C) e caídos junto ao pasto. O plantel era formado por 70 ovinos, entre eles, fêmeas, machos e cordeiros. Destes, 11 adoeceram e morreram. Os ovinos eram recolhidos durante a noite para um abrigo e a doença era observada pela manhã, no momento em que estes animais eram soltos e movimentados. Os sinais clínicos consistiam de incoordenação motora, com dificuldade em manter-se em estação, contrações bruscas com queda no chão, salivação, opistótono seguido de morte rápida ou morte algumas horas após o início dos sinais clínicos. Em alguns casos, os sinais clínicos eram desencadeados apenas quando os ovinos eram movimentados. Dois ovinos dessa propriedade foram necropsiados, porém não foram observadas lesões macroscópicas ou microscópicas significativas. Depois de estabelecido a suspeita de intoxicação pelos frutos de E. deciduum, as árvores foram cortadas pelo proprietário e os ovinos foram retirados do local. Aproximadamente 40 dias após, no mês de março, os ovinos foram reintroduzidos no mesmo local e mais cinco ovinos morreram apresentando os mesmos sinais clínicos observados na suspeita de intoxicação pelos frutos de E. deciduum. Ao observar o local onde os ovinos se encontravam, verificou-se que nos troncos das árvores de "cocão" anteriormente cortados, havia muitos brotos (Fig.1D), os quais tinham evidencias de terem sido consumidos. O Surto 2 ocorreu no mês de dezembro de 2008. O proprietário informou que no passado tinha criação de ovinos, mas substituiu os ovinos por bovinos, pois, o número de ovinos que morriam com sintomatologia nervosa era muito alto. Em 2008, retornou 

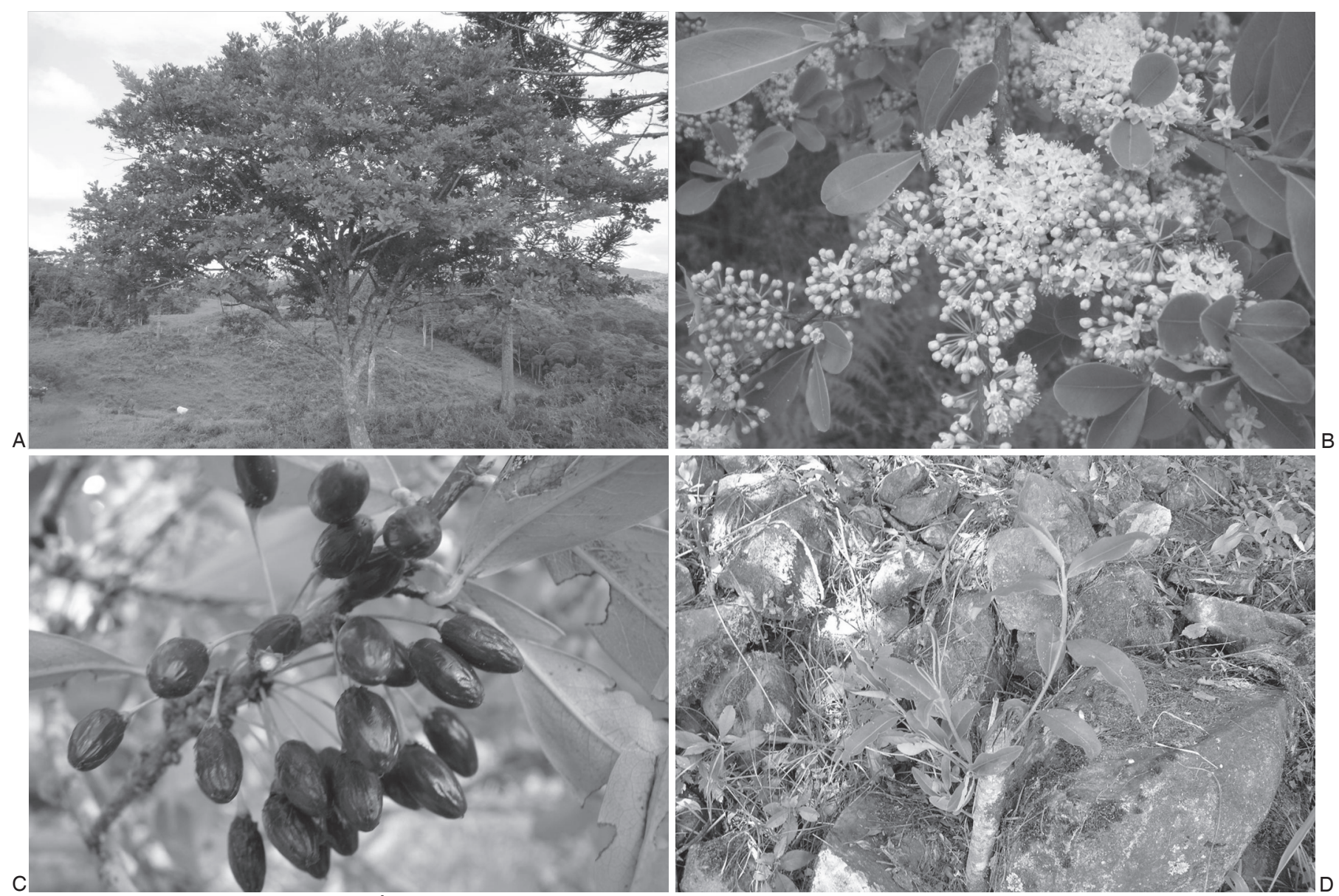

Fig.1. Erythroxylum deciduum. (A) Árvore adulta. (B) Estágio de floração. (C) Estágio de frutificação. (D) Brotação no tronco da árvore.

a criação de ovinos e a doença tornou a acontecer, com maior mortalidade nos meses de outubro e dezembro. Os ovinos permaneciam em área constituída por campo nativo e mata, sendo que E. deciduum era a árvore observada com freqüência . Neste surto, no mês de dezembro, E. deciduum encontrava-se no início da frutificação com frutos verdes e havia grande quantidade de troncos da planta, com brotação na beira da mata, em local que anteriormente tinha sido roçado. Em todas as brotações, havia sinal de consumo das folhas e, em algumas, foi observado lã presa nos galhos. O plantel era constituído por 160 ovinos, entre fêmeas, machos e cordeiros. Destes, 15 adoeceram e morreram. A doença ocorria quando os ovinos eram recolhidos ao final da tarde para um abrigo, no momento em que estes eram movimentados. Os sinais observados por ele foram enrijecimento das pernas, salivação, respiração acelerada, quedas e morte rápida. Um ovino dessa propriedade foi necropsiado pelo veterinário de campo, que observou folhas intactas e maceradas de $E$. deciduum nos pré-estômagos.

\section{Intoxicação experimental}

Sinais clínicos. Os principais sinais clínicos foram: depressão, sonolência e quando os animais eram movimentados manifestavam incoordenação motora, desequilíbrio, arraste de pinças, quedas, dificuldade para se le-

vantar e voltar à estação (Fig.2A) e, quando em estação permaneciam com os membros abertos. Também foi observado: salivação intensa (Fig.2B), regurgitação, bruxismo, aumento das freqüências cardíacas e respiratórias. Em um animal que foi realizado eletrocardiograma foi constatado taquicardia, arritmia e fibrilação.

Resumos dos protocolos dos experimentos. O Ovino 1 , que recebeu $10 \mathrm{~g} / \mathrm{kg}$ de folhas de brotação de troncos de E. deciduum, colhidas no mês de março de 2006, foi encontrado morto 12 horas após a administração da planta.

O Ovino 9, que recebeu $10 \mathrm{~g} / \mathrm{kg}$ de folhas jovens de árvore, coletadas no final de março de 2008 e Ovino 11, que recebeu $15 \mathrm{~g} / \mathrm{kg}$ de folhas adultas colhidas no mês de junho de 2008, apresentaram sonolência, taquicardia e salivação moderadas e 12 horas após estavam recuperados.

O Ovino 7, que recebeu folhas jovens de árvore, na dose de $15 \mathrm{~g} / \mathrm{kg}$, colhidas no mês de março de 2008 , apresentou sinais clínicos uma hora após o término do fornecimento. Verificaram-se sonolência, tontura, andar cambaleante, salivação intensa, regurgitação e bruxismo. Quando em estação, permanecia com os membros abertos. Ao ser movimentado, o animal apresentava incoordenação motora com andar cambaleante, tropeços e quedas com tentativa, sem êxito, de se levantar. Os sinais se agrava- 

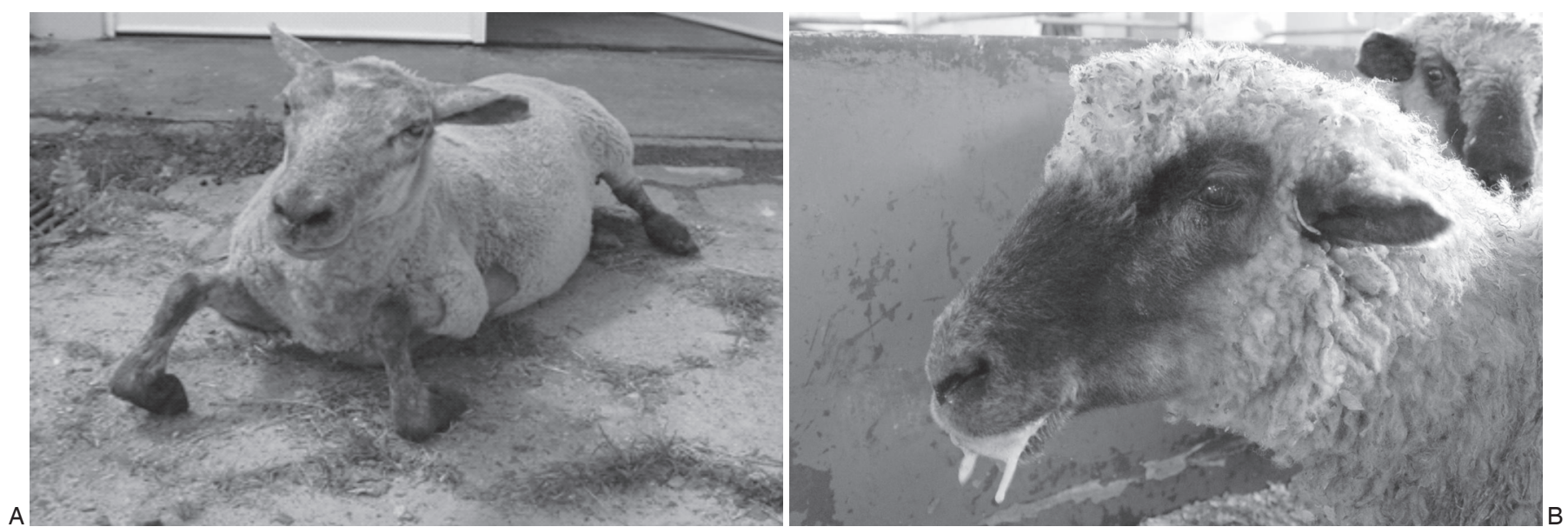

Fig.2. Intoxicação experimental com Erythroxylum deciduum, sinais clínicos. (A) Quedas com tentativas para se levantar. (B) Ovino com salivação intensa.

vam quando o animal era forçado a caminhar ou quando era movimentado. Três horas após o início dos sinais clínicos, o ovino apresentou decúbito lateral, movimentos de pedalagem, seguido de morte em alguns segundos.

O Ovino 8, que recebeu folhas jovens de árvore na dose de $15 \mathrm{~g} / \mathrm{kg}$ colhidas no mês de março de 2008 , imediatamente após a administração, apresentou salivação intensa, andar cambaleante, arrastando as pinças anteriores e posteriores, quedas, regurgitação, bruxismo, dispnéia e intensa taquicardia. $\mathrm{O}$ animal ficou em observação por um período de 12 horas. Durante este período, permaneceu em estação com salivação intensa e bruxismo constante. Após, apresentou sonolência, porém permanecia atento a ruídos. Ao movimentar-se, manifestou tontura, queda, movimentos de pedalagem, seguido de morte.

O ovino 10, que recebeu folhas jovens de árvore, na dose de $15 \mathrm{~g} / \mathrm{kg}$, colhidas no mês de março de 2008 , no exame eletrocardiográfico, apresentou dispneia, taquicardia, arritmia e fibrilação seguida de morte 10 minutos após o início dos sinais clínicos.

O Ovino 15, que recebeu folhas em brotação de troncos na dose de $15 \mathrm{~g} / \mathrm{kg}$, colhidas no mês de dezembro de 2008, duas horas após a administração, ao caminhar, apresentou rigidez dos membros, arraste das pinças anteriores e posteriores, quedas com permanência dos membros esticados, posições atípicas, tentativa de levantar, bruxismo, dispnéia e intensa taquicardia. A maior parte do tempo permaneceu em estação, com a cabeça baixa e com os membros abertos, evitando a movimentação. Apresentou bruxismo constante, sonolência, ficando atento a movimentos e ruídos e, em certos momentos, alimentavase. Morreu 18 horas após o início dos sinais clínicos.

O Ovino 16, que recebeu frutos maduros na dose de $15 \mathrm{~g} / \mathrm{kg}$, colhidas no mês de janeiro de 2009 , duas horas após a administração, pastava normalmente quando estimulado a caminhar, teve várias quedas com permanência dos membros esticados e tentativa de se levantar, bruxismo, taquicardia e dificuldade respiratória. Morreu 30 minutos após o início dos sinais clínicos.
Os ovinos que receberam folhas adultas colhidas no mês de agosto de 2007, nas doses de $5 \mathrm{~g} / \mathrm{kg}$ (Ovino 2), $7,5 \mathrm{~g} / \mathrm{kg}$ (Ovino 3), 10g/kg (Ovino 4); folhas jovens de árvores colhidas no mês de outubro de 2007, nas doses de $5 \mathrm{~g} /$ $\mathrm{kg}$ (Ovino 5) e 20 $/ \mathrm{kg}$ (Ovino 6); folhas adultas colhidas no mês de junho de 2008, na dose de $15 \mathrm{~g} / \mathrm{kg}$ (Ovino 12) e folhas adultas colhidas no mês de setembro de 2008, nas doses de $15 \mathrm{~g} / \mathrm{kg}$ (Ovino 13) e 20 g/kg (Ovino 14), não apresentaram alterações clínicas.

Lesões macro e microscópicas. Os ovinos que morreram (Ovinos 1, 7, 8, 10, 15 e 16) apresentaram somente achados macroscópicos inespecíficos caracterizados por petéquias multifocais no baço e hiperemia na mucosa da traquéia e sistema nervoso. Ovino16 tinha hemorragias multifocais no timo. Em todos os ovinos, não foram observadas lesões microscópicas significativas.

\section{DISCUSSÃO}

Os fatores predisponentes para a intoxicação de Erythroxylum deciduum foram a ocorrência de brotações nos troncos das árvores cortadas, associados à baixa disponibilidade de pastagem e à disponibilidade de frutos maduros caídos junto à pastagem. Segundo Colodel et al. (2004), os ovinos consomem com avidez os frutos maduros de cocão na pastagem. Porém, no levantamento realizado, um produtor observou que alguns animais evitam o consumo, justificando o motivo para que muitos animais não adoecessem.

A taxa de morbidade obtida através de informações de produtores foi de $25 \%$ e dos dois surtos avaliados no município de Rancho Queimado foi de 22,85 e 9,38\%. A taxa de mortalidade foi próxima de $100 \%$ em ambos os surtos.

Experimentalmente, folhas de E. deciduum coletadas de árvores adultas e de brotação de troncos, nos meses de dezembro e março foram letais nas doses de 10 e $15 \mathrm{~g} /$ $\mathrm{kg}$, e equivalem, a toxidade dos frutos reproduzida experimentalmente no presente estudo e dos frutos de $E$. argentinum, obtida por Barros et al. (2004).

As folhas coletadas nos meses de junho, agosto, setembro e outubro nas doses de até $20 \mathrm{~g} / \mathrm{kg}$ produziram ape- 
nas alterações clínicas leves nos Ovinos 9 e 11, caracterizando menor toxidade da planta nessa época do ano. Esse fato associado a pouca quantidade de folhas encontradas nas plantas entre o final do outono até o início da primavera contribui para a não ocorrência da enfermidade neste período.

O quadro clínico e a ausência de lesões anatomohistopatológicas significativas, observadas na intoxicação espontânea e experimental pelas folhas e frutos de $E$. deciduum em ovinos, no estado de Santa Catarina, foram semelhantes aos descritos nos surtos de intoxicação pelos frutos dessa planta no estado do Rio Grande do Sul, descritos por Barros et al. (2004) e Colodel et al. (2004).

Os principais sinais clínicos observados foram depressão, sonolência, incoordenação motora, desequilíbrio, quedas, dificuldade para levantar e estação com os membros abertos. Esses sinais eram exacerbados após exercícios. No Brasil existem outras plantas tóxicas que produzem sinais nervosos em ovinos e que também não apresentam alterações macroscópicas importantes e devem ser levadas em consideração para realização de diagnóstico diferencial. Dentre elas, citam-se: intoxicação por Halimium brasiliense, que ocorre no Rio Grande do Sul, entre os meses de agosto e novembro e possui quadro clínico epileptiforme periódico e são descritas degeneração axonal e acúmulo de lipofuscina nos neurônios e macrófagos de diversos órgãos (Riet-Correa et al. 1998); Marsdenia spp. (mata calado), que podem afetar ovinos e bovinos, ocorre na região semi-árida da Paraíba e Rio Grande do Norte e também não são descritas alterações macro e microscópicas (Riet-Correa et al. 2004); intoxicação por Turbina cordata, Ipomoea carnea, I. sericophylla, I. riedelii e I. asarifolia que afeta principalmente caprinos e ovinos, mas também bovinos e búfalos e ocorrem com maior frequência no nordeste. Os sinais clínicos se caracterizam por tremores de cabeça e, histologicamente, são observados vacúolos no citoplasma dos neurônios de Purkinje (Barbosa et al. 2005, Barbosa et al. 2006, Dantas et al. 2006, Armién et al. 2007).

O diagnóstico de intoxicação por folhas e frutos de $E$. deciduum baseou-se nos dados epidemiológicos, sinais clínicos, ausência de lesões macro e microscópicas significativas, presença da planta no local e reprodução experimental.

\section{CONCLUSÕES}

As folhas de brotação de troncos de árvores Erythroxylum deciduum ingeridas pelos ovinos nos meses de dezembro e março foram letais, em doses únicas de 10 e $15 \mathrm{~g} / \mathrm{kg}$, respectivamente.

Folhas de E. deciduum coletadas no nos meses de junho, agosto, setembro e outubro não foram letais para ovinos, nas doses de até $20 \mathrm{~g} / \mathrm{kg}$, demonstrando menor toxidade nesta época do ano.
Os frutos de E. deciduum foram tóxicos para o único ovino a que foi administrado, na dose de $15 \mathrm{~g} / \mathrm{kg}$ e sua toxicidade equivale a toxicidade das folhas ingeridas no final da primavera e verão.

Surtos de doença em ovinos com alterações nervosas, que ocorrem no final da primavera e verão, no estado de Santa Catarina, podem ser atribuídos a intoxicação pelos frutos e ou, pelas folhas de E. deciduum.

Agradecimentos.- À CAPES pela concessão de bolsa de mestrado, à Universidade do Estado de Santa Catarina (UDESC) pelo apoio financeiro ao projeto, à professora Roseli Lopes Bortulizzi pela classificação botânica, e ao encarregado de fazenda (Surto 1) José Broering.

\section{REFERÊNCIAS}

Armién A.G., Tokarnia C.H., Peixoto P.V. \& Frese K. 2007. Spontaneous and experimental glycoprotein storage disease of goats induced by Ipomoea carnea subsp. fistulosa (Convolvulaceae). Vet. Pathol. 44:170-184.

Aynilian G.H., Duke J.A., Gentner W.A. \& Farnsworth N.R. 1974. Cocaine content of Erythroxylum species. J. Pharm. Sci. 63(12):19381939.

Barbosa J.D., Oliveira C.M.C., Duarte M.D., Peixoto P.V. \& Tokarnia C.H. 2005. Intoxicações experimental e natural por Ipomoea asarifolia (Convolvulaceae) em búfalos e outros ruminantes. Pesq. Vet. Bras. 25(4):231-234

Barbosa R.C., Riet-Correa F., Medeiros R.T.M., Lima E.F., Barros S.S., Gimeno E.R., Molyneux R.J. \& Gardner D.R. 2006. Intoxication by Ipomoea sericophylla and Ipomoea riedelii in goats in the state of Paraíba, northestern Brazil. Toxicon 47:371-379.

Barros R.R., Teixeira F.R., Oliveira F.N., Rissi D.R., Rech R.R. \& Barros C.S.L. 2004. Poisoning in sheep from the ingestion of fruits Erythroxylum argentinum. Vet. Human Toxicol. 46:173-175.

Colodel E.M., Seitz A.L., Schmitz M., Borba M.R., Raymundo D.L. \& Driemeier D. 2004. Intoxicação por Erythroxylum deciduum em ovinos. Pesq. Vet. Bras. 24(3):165-168.

Dantas A.F.M., Riet-Correa F., Gardner D.R., Medeiros R.M.T., Barros S.S., Anjos B.L. \& Lucena R.B. 2006. Swainsonine induced lysosomal storage disease in goats caused by the ingestion of Turbina cordata in northeastern Brazil. Toxicon 49(1):111-116.

Lorenzi H. 2002. Árvores Brasileiras. $2^{\mathrm{a}}$ ed. Instituto Plantarum, Nova Odessa, SP, p.107.

Prophet E.B., Mills B., Arrington J.B. \& Sobin L.H. 1992. Laboratory Methods in Histotechnology. Armed Forces Institute of Pathology, Washington, DC. 279p.

Riet-correa F., Schild A.L. \& Fernandes C.G. 1998. Enfermidades do sistema nervoso dos ruminantes no sul do Rio Grande do Sul. Ciência Rural 28(2):341-348.

Riet-Correa F., Silva D.M., Oliveira O.F., Simões S.V.D., Medeiros R.M.T. \& Nóbrega R.S. 2004. Intoxicação por Marsdenia spp (matacalado) em ruminantes. Pesq. Vet. Bras. 24(Supl.):50-51.

Sobral M. 1987. Erythroxylum (Erythroxylaceae) no Rio Grande do Sul, Brasil. Pesq. Bot. 38:7-42.

Zuanazzi J.A., Tremea V., Limberger R.P., Sobral M. \& Henriques Amélia T. 2001. Alkaloids of Erythroxylum (Erythroxylaceae) species from Southern Brazil. Biochem. Sys. Ecol. 29:819-825. 